Acta Hispanica (2020) Supplementum II: 923-932

\title{
LA MUJER Y EL PAPEL DE LA UNIVERSIDAD COLOMBIANA HACIA MEDIADOS DEL SIGLO XX
}

\author{
Lina Adriana Parra BáEz
}

\author{
Universidad Pedagógica y Tecnológica de Colombia
}

\begin{abstract}
Resumen: En Colombia, hacia mediados del siglo XX, la universidad tuvo una enorme responsabilidad frente a los diversos hechos y fenómenos sociales que se fueron presentando. Por ello, se esperó que contribuyera a la solución de los diferentes conflictos y problemas nacionales que amenazaban de diversa manera la estabilidad del Estado, a partir de situaciones de violencia política y crisis económica. Aquí se dirigía la postura: "ante este ciclón que amenaza, la universidad está llamada a rescatar a la nación de tan triste situación [...] El analfabetismo crónico en Colombia ha permitido este desequilibrio, y si no existe preocupación alguna por semejante falla, el camino hacia el desorden es inmediato. Solo a través de la educación y de las energías y dineros que a ella -la educación- se consagren puede aliviarse tal estado de cosas" "Educación de la Juventud", 1954). En ese sentido, la mujer estuvo llamada a reconocer su papel a través de respuestas a los retos planteados, con miras a generar procesos de desarrollo para la generación de nuevos conocimientos.
\end{abstract}

Palabras clave: universidad, mujer, educación.

\begin{abstract}
In Colombia, towards the middle of the 20th century, the university had an enormous responsibility in the face of the various social events and phenomena that were presented. Therefore, it was hoped that it would contribute to the solution of the different national conflicts and problems that threatened in a different way the stability of the State, based on situations of political violence and economic crisis. Here the position was directed: "in the face of this cyclone that threatens, the university is called to rescue the nation from such a sad situation [...] Chronic illiteracy in Colombia has allowed this imbalance, and if there is no concern for such a failure, the road to The disorder is immediate. Only through education and the energies and money that are dedicated to it -education - can this state of affairs be relieved". In that sense, women were called to recognize their role through responses to the challenges posed, with a view to generating development processes for the generation of new knowledge.
\end{abstract}

Keywords: University, Women, Education.

\section{Antecedentes}

Hablar de enseñanza superior en Colombia, es remitirnos al siglo XVI con la creación de una Universidad Dominicana. Este establecimiento y las otras tres instituciones de enseñanza superior, igualmente religiosas, que funcionaron en el país, en la época de la colonia, se dedicaron casi exclusivamente a la enseñanza de la teología, la filosofía, las letras y el derecho (Le Bot, 1985: 72-73). A finales del siglo XIX empezaron a surgir en Colombia las universidades que brindaban a sus estudiantes las carreras liberales, que en 
La mujer y el papel de la universidad colombiana hacia mediados del siglo XX

principio solo pudieron ser cursadas por varones. Y solo a mediados del siglo XX, fue cuando las mujeres tuvieron la opción de seguir algunas de ellas, después de librar largas batallas.

A finales de la década de los años cuarenta y comienzos del cincuenta del siglo XX, surgieron varias universidades oficiales y privadas en el país como la Universidad de los Andes, Universidad Industrial de Santander y la Universidad del Valle, entre otras, las cuales cumplieron tareas de gran trascendencia en procesos de desarrollo cultural, social y científico del país.

En ese sentido, la universidad empezó a considerarse como un lugar en donde se realizaban los estudios superiores, donde se formaban los nuevos espíritus, se impulsaba la investigación como medio de generación de conocimiento, y era allí, en donde se debían fortalecer los valores morales, cívicos y ciudadanos. Es decir, la responsabilidad de los centros de enseñanza superior se direccionaba hacia el progreso de la nación. Por tanto, la misión de la Universidad Colombiana fue amplia y trascendente en el campo del conocimiento y de otras tareas sociales, lo cual era muy significativo para un país como el nuestro, porque esto implicaba gran responsabilidad en todo sentido y de manera particular, el impulso a la investigación, como bien lo dijera José María Cháves en el informe del Fondo Universitario Nacional en el año 1957 que anotaba, entre muchos otros aspectos: "la Universidad como centro de estudios y núcleo de científicos tiene como misión investigadora; como escuela y maestra tiene una misión educativa; y como entidad autónoma interesada en el progreso humano tiene una misión social" (Chaves, 1957: 15-16).

Por ello, como afirma Chaves (1957: 7) podríamos decir que las tendencias de la educación en Colombia hacia la mitad de la década de los años 50 del siglo XX, manifestadas en recomendaciones de misiones extranjeras como el BIRF, CURRIE, LEBRET, CHAILLOUX, la CEPAL y la UNESCO, se orientaron a que el Ministerio de Educación Nacional fuera el ente rector en lo relativo a planeamiento, control y coordinación de la enseñanza. El Ministerio de Hacienda en materia de presupuesto y obtención de recursos, y la industria en su vinculación con la universidad.

Así, la enseñanza técnica fue una de las preferencias de la universidad dada la expansión industrial del país, pues surgieron nuevas relaciones de producción, y por supuesto, el adelanto tecnológico que requería de las instituciones superiores y por ende de profesionales preparados, lo cual significaba la entrada de la universidad a la modernidad. Sin embargo, las disciplinas humanistas marcaban el nivel cultural de los individuos y de los pueblos, por lo cual debieron mantenerse e incluso ampliarse.

Pero como fue necesario crear nuevas formas de vida, de comunicarse, de abrir cada día nuevos horizontes para la creatividad y el esfuerzo de las gentes, requirieron del desarrollo de las ciencias exactas cuyo objetivo fue el conocimiento de la naturaleza y el aprovechamiento de los recursos en bien de la humanidad (Chaves, 1957: 8). Total, era necesario e inevitable la formación de profesionales en distintas disciplinas, por lo cual 
fue urgente que en los planes de estudio de las carreras técnicas no se olvidara la parte humanística.

Es importante resaltar los lineamientos de una filosofía educativa del país dentro de una pedagogía integral que armonizara la concepción cristiana católica con la estructura del Estado que caracterizara el espíritu nacional. Así la educación superior sin perder su fisonomía humanística debió atender a la formación científica y tecnológica para que las nuevas generaciones fuesen capaces de atender los problemas de orden técnico, económico y social de la modernidad.

Particularmente, la expectativa social de la universidad, en la época del gobierno de Rojas Pinilla, llevó a concebir reformas importantes que debieron orientarse hacia una política de admisión, la especialización técnica, la unificación de pénsumes mínimos y el fomento de la investigación científica como base de la docencia, tal como lo señala Chaves (1957: 27).

En cuanto a la selección de aspirantes se acordó hacer estudios para que solo fueran admitidos estudiantes que aseguraran su permanencia en las facultades y consideraban que era para aquellas o aquellos que supieran aprovechar el tiempo, por lo cual cada programa debía adoptar un sistema riguroso de exigencia para los estudiantes de manera que manifestaran estar dispuestos a finalizar la carrera. Así para el nivel de posgraduados, con el fin de adelantar una especialización en el exterior, se ofrecía respaldo a través del ICETEX.

Respecto a la unificación de pénsumes universitarios, el Fondo Universitario Nacional, fue adelantando seminarios nacionales de las distintas carreras, los cuales les permitieron conocer aspectos programáticos de cada universidad y adelantar debates para unificar criterios y contenidos mínimos. En el año de 1957 ya se habían registrado las conclusiones de seminarios nacionales de ciencias sociales, medicina, ingeniería, física, matemáticas, veterinaria, educación física, filología y letras, odontología, economía, arquitectura, enseñanza de la pedagogía y otras (Chaves 1957: 105).

De igual manera, para Chaves (1957: 28) el incremento de la investigación científica se tuvo en cuenta como un punto trascendental dado que existía una responsabilidad moral, científica y social muy grande. Las universidades colombianas en ese momento, con el apoyo del Fondo Universitario Nacional, debieron presentar proyectos para recibir recursos de fundaciones norteamericanas como la Rockefeller y Kellogg, además de la fundación iberoamericana de Hamburgo.

Las exigencias a las universidades incluían lo que se imponía en expansión del conocimiento y el desarrollo de técnicas sobre la base del conocimiento de elevado nivel para que en el futuro se convirtieran en tecnologías respetables. Las universidades debieron asumir como meta, como principio y como fin, el justificar su propia función como parte del contexto social.

Las diferentes ideas y corrientes de pensamiento de mayor trascendencia se convirtieron en medio y en fuerza para definir políticas educativas y pedagógicas en la educación de ciudadanas y ciudadanos colombianos. De manera concreta fueron 
La mujer y el papel de la universidad colombiana hacia mediados del siglo XX

esenciales en el establecimiento de currículos para la formación de las maestras universitarias. Por eso, al observar los planes de estudio, no nos podemos sorprender con la enseñanza religiosa, con los principios cívicos, con la creación de especializaciones y doctorados sobre el trabajo investigativo, entre otros aspectos.

Por esa razón, se entiende que la formación de las maestras en las instituciones femeninas se orientó hacia la consolidación de un modelo educativo de corte religioso, familiar, patriótico y social que contribuyera a contrarrestar los avances del comunismo y el socialismo en el país y a que se fortalecieran los principios y valores morales y ciudadanos.

En el caso particular de la educación femenina para el ejercicio docente, se observa de manera especial a la Universidad Pedagógica Nacional Femenina que creó una estructura académica desde la concepción de diferentes niveles de enseñanza: universidad con cinco facultades, el instituto de bachillerato, el instituto Pedagógico Nacional, las escuelas primarias anexas, el instituto de educación preescolar y el kindergarten que se articularon y funcionaron de manera sistemática y eficaz. Allí se desarrolló la labor pedagógica y se fueron formando grupos de niñas y jóvenes como maestras en los distintos niveles educativos (Muller de Ceballos, 1992: 113-114).

Se podría afirmar que, durante la administración de Rojas Pinilla, la educación superior femenina se guio por los principios religiosos - católicos porque ante todo se buscaba formar una mujer que pudiera sostener un hogar cristiano y fuera capaz de ayudar a construir un país con valores cívicos y morales. Se aspiró que, a través de las enseñanzas de la vida cristiana, de ritos y prácticas religiosas, junto a programas de proyección a la comunidad del entorno social y al lado del apostolado de la Iglesia Católica, unieran principios de patriotismo y contribuyeran a hacer del país el mejor espacio para crear, compartir y vivir dignamente. Por esa razón, Rojas Pinilla atacó, de una u otra forma, todo lo relacionado con el comunismo, y por tanto con el marxismo que era su filosofía oficial, ya que, como doctrina y teoría social, económica y política, basada en la obra de Karl Marx y sus seguidores, indisolublemente unida a dos ideologías y movimientos políticos: el socialismo y el comunismo, podían alejar a la mujer de su verdadero ser, de su esencia y papel en la sociedad.

También porque el marxismo consideraba a la religión como el opio del pueblo. Además, para Marx no existía Dios sino la materia como única realidad, representada en la misma esencia del hombre; por tanto, la educación como actividad estrechamente unida a la aptitud para el trabajo productivo del sujeto educado, era la forma de llegar al verdadero humanismo. Recordemos que Rojas era ferviente católico, amigo de la Iglesia y fiel seguidor de los postulados del Papa. Él pensaba que la educación colombiana era hija de la Iglesia, y era a ella a quien le correspondía formar el espíritu de las nuevas generaciones, sin dejar de lado el amor e identidad a la Patria, a lo nuestro, a nuestras raíces. 


\section{El papel de la universidad colombiana a mediados del siglo $\mathrm{XX}$}

El teórico Jaime Posada en su libro "Universidad, democracia y país”, expresaba que la universidad estaba llamada a rescatar a la nación de la situación social que vivía por aquella época, debido al notable analfabetismo que se presentaba en Colombia y que había llevado al país a un gran desequilibrio. Solo la educación sería capaz de aliviar esa situación, y agregaba: "si la educación quiere ser limpia y luminosa, estimular la formación de la personalidad y adiestrar para el desempeño en comunidad, tiene que ser una educación para la libertad y la democracia" (Posada, 1957: 122).

Con ello, la Universidad buscaba hacer de sus alumnos, hombres y mujeres honrados, capaces de crear, imaginar, innovar y establecer un ambiente de comprensión con ideas propias y alto sentido de responsabilidad, aptos para cimentar democracia social, integral y constructiva. Futuros profesionales, capaces de comprender el mundo que los rodeaba, por el conocimiento de sus problemas y el ambiente en el que estaban inmersos que era el propio y con posibilidades de resignificar su identidad cultural.

Lo anterior significaba oportunidades de perfeccionamiento y de servicio a los demás seres humanos que estaba incluido en los propósitos de la universidad, pues como lo anotara el autor José María Chaves:

a ella corresponde el estudio de nuestros problemas nacionales y la búsqueda de soluciones a ellos apropiadas. El hacer consciente a los jóvenes de los problemas y el enseñarles que la solución de las cuestiones sociales depende en gran manera de la buena voluntad que tengan los hombres en resolverlos. En nuestra civilización peculiar la preparación social que una universidad como ésta debe dar a sus alumnos ha de ser profesional y técnica y al mismo tiempo humanística (1957: 17).

La formación profesional tenía como eje la investigación científica, para lo cual se exigía diversos recursos educativos para cuyas diferentes actividades era importante apropiarse de estudios de los pueblos, especialmente en cuanto a su historia y geografía, con el propósito de mejorar las condiciones de los ciudadanos y el futuro de las comunidades. Esta fue una acción fundamental para la Universidad en Colombia porque emprendió la creación de una conciencia social en sus educandos. Así mismo, la construcción y conservación de un ambiente de libertad fue uno de sus papeles en la vida nacional.

En una de las alocuciones del presidente Gustavo Rojas Pinilla en el año 1954, al referirse al papel que debía cumplir la Universidad bajo su gobierno y para armonizar la vida del pueblo con los ideales nacionales, decía: 
La mujer y el papel de la universidad colombiana hacia mediados del siglo XX

tenemos que hacer de la universidad entidad rectora de nuestra comunidad de creencias, sentimientos y aspiraciones. Pues si nos hemos trazado desde el nacimiento de la República una meta de creaciones democráticas, la Universidad tiene que aportar sus valores en servicio de esos ideales, y está en la obligación de averiguar cuáles son los medios adecuados para alcanzarla [...] La Universidad, además de la formación intelectual, moral y física de sus educandos, debe extender su influencia al ámbito social; su misión debe ser de siembra y penetración en todas las esferas sociales. Ella debe propender por popularizar la cultura, porque de lo contrario, si la Universidad no irradia, se muere. Sacudamos la arquitectura universitaria para despojarla de cuanto le sea incómodo y no refleje su espíritu; introduzcamos en sus claustros todas las vivencias nacionales que contengan acervos de cultura, justicia, trabajo, progreso y esperanza. Avivemos en el espíritu de la juventud universitaria la misión secular que el porvenir le depara, para que entienda que en ella reside la eternidad de la Patria (Rojas Pinilla, 1954a: 136).

Esta idea de Gustavo Rojas Pinilla se plasmó en el Decreto Ley No. 3386 del 22 de diciembre de 1954, mediante el cual se creó el Fondo Universitario Nacional, institución descentralizada con el fin de desarrollar actividades de integración entre las universidades; contratar profesores extranjeros especializados; organizar postgrados; de distribuir recursos económicos, asignados por el Estado, entre las Universidades públicas y privadas; y de impulsar la investigación científica.

En general, el periodo del gobierno de Rojas Pinilla fue trascendental para el fortalecimiento de la enseñanza superior, en la búsqueda de ampliar los roles universitarios y de concretar acciones en beneficio de la Patria. Aunque algunos críticos no vieron con buenos ojos la intervención de la Universidad Colombiana por parte del Estado, y el mayor enojo para algunos sectores, fue la representación que se le dio a la Iglesia en los Consejos Directivos. El presidente consideraba que la Universidad no podía estar ausente de los grandes debates nacionales, pues la veía como una entidad burocratizada y dedicada a fabricar profesionales sin sentido social, sin capacidad de investigación y sin estructura moral. De ahí que en uno de sus discursos dijera:

la Universidad tiene que vivir en contacto permanente con la realidad del país, siempre lista a preparar los profesionales que nuestro progreso demande. Pero por, sobre todo, debe estructurar moralmente a sus estudiantes, enseñarles que la ciencia no ha sido acumulada en paciente y heroica brega de generaciones para que sirva como instrumento de enriquecimiento y explotación (Rojas Pinilla, 1954c: 168). 
Esta expresión significaba que la Universidad tenía un fin más alto que era ayudar y mejorar la condición de vida de las personas que no pudieran ingresar a las aulas universitarias. Así se estaba fortaleciendo el sentido social de las nuevas generaciones de egresados de la universidad, con lo cual se aspiraba a trabajar para mantener la paz, la justicia social y la libertad de los colombianos.

En esas circunstancias, la universidad colombiana tuvo que cumplir un papel preponderante en el contexto nacional desde una perspectiva de humanización y el cumplimiento de la búsqueda dinámica y constante del conocimiento bajo el horizonte de la investigación científica, unida a la ética y al incremento de estudios de humanidades

y ciencias sociales que le permitieron alcanzar calidad en la enseñanza y cierta autonomía en los diferentes campos del saber.

De otro lado, el hecho de abrir las puertas de la enseñanza superior a la mujer, la universidad estaba cumpliendo con un papel importante en la vida nacional y significó un acontecimiento revolucionario que tuvo impacto en toda la sociedad. No se podía desconocer que la educación como fuente de progreso abría enormes posibilidades a la mujer y le daba la capacidad de afrontar riesgos. Desde ese momento, todo fue cambiando y las castas féminas fueron asumiendo roles distintos que fueron representado cambios culturales significativos para el país.

Por ello, la concepción sobre la formación de la mujer dentro de un sistema educativo universitario en Colombia se relaciona con su aceptación e ingreso a instituciones de enseñanza superior. Este aspecto se puede entender a partir del año 1945 cuando en este año se promulgó la ley 48 que creaba en el país los colegios mayores femeninos que eran establecimientos dedicados a formar en especializaciones como letras, delineantes de arquitectura, secretariado, servicio social, periodismo, auxiliares de cirugía, orientación familiar y otras.

\section{Mujer y educación superior en Colombia}

Desde el año 1940 funcionaban los colegios nacionales femeninos de bachillerato como el liceo nacional femenino "Antonia Santos” de Bogotá y el de Zipaquirá que ya gozaban de gran prestigio. De igual manera, los colegios departamentales femeninos como el centro educacional femenino de Antioquia - CEFA que tuvo varios nombres: Instituto central femenino y luego Instituto Isabel la católica que llegó a matricular 4.000 alumnas y contó con las secciones: normalista, de bachillerato, comercio y distintas carreras intermedias. Así, también la existencia de escuelas normales femeninas tanto departamentales como nacionales que contribuyeron de manera notable a orientar y engrandecer la educación de la mujer en nuestra República (Gaviria, 1967: 187-189).

Estos programas permitieron abrir diferentes campos a la mujer e impactar social y culturalmente en distintas regiones del país. Este fue el punto de partida para iniciar la formación y preparación sistemática de la mujer con el fin de evitar que se quedara solo con la escuela primaria y aun la secundaria, sino que se adentrara en lo profesional. El 
La mujer y el papel de la universidad colombiana hacia mediados del siglo XX

reto era muy grande y fue la oportunidad para escalar posiciones y participar en diferentes actividades e incluso, en decisiones del Estado.

La vida moderna trajo nuevas preocupaciones e inquietudes a la mujer, así como nuevos derechos y deberes. Por eso, irrumpió en el comercio, la economía, la pedagogía, la política, la cultura y la industria, en las oficinas públicas y privadas, fue ocupando mejores lugares, aunque de alguna manera afectaba la vida familiar (Rodríguez Rojas, 1963: 281).

La misión de la mujer en el campo educativo superior la asumió principalmente con base en su formación de maestra para la enseñanza superior. La Universidad Pedagógica Femenina Nacional fue la encargada de formar las maestras universitarias en las especialidades de: pedagogía; biología y química; física y matemáticas; etnología; ciencias sociales y económicas; filología e idiomas y educación física ("Noticias de la Universidad", 1953: 36).

La generación de mujeres formadas allí, en la época de la dictadura militar, se ubicaron y destacaron en universidades públicas y privadas, en los distintos campos del saber, mostrando capacidad y entusiasmo en importantes responsabilidades en los campos de la docencia y la investigación.

En los planes de estudio de cada especialidad, además de las materias propias de su área disciplinar, se ofreció con sumo cuidado la pedagogía, las prácticas docentes, idiomas extranjeros, prácticas cívicas y cultura religiosa cada una con su particular objetivo. Esta última, fue porque ese era el querer del gobierno, como lo manifestaba en sus discursos y mensajes en distintos escenarios el presidente Rojas. Por ejemplo, en el Huila en una manifestación popular el día 7 de marzo de 1954, al referirse a la fe religiosa, decía:

convencido como estoy de que es necesario saturar las mentes y los corazones de todos los hijos de Colombia con los principios que son soporte de la paz, de la justicia y de la libertad, vuelvo hoy a repetir que solo en la doctrina de Cristo pueden hallarse las bases de la recta moral y solo marchando tras las banderas nacionales de Bolívar puede llegarse a la consolidación de la grandeza patria. Cristo y Bolívar en su más santo y patriótico concepto, son postulados luminosos que guían y unen a todos los ciudadanos, porque en ellos se confunden unas mismas creencias religiosas y la misma veneración por quien nos dio libertad y estructuró la grandeza de la República, luchando hasta en los postreros instantes de su agonía porque cesaran los odios de partido y se consolidara la unión. Como símbolos perfectos de Dios y de la Patria, estos dos postulados, tan genuinamente nacionales, orientan la acción del Gobierno en si invariable propósito de buscar el bien espiritual y material de todos los colombianos (Rojas Pinilla, 1954b: 45-46). 
El gobierno sentaba allí una política de Estado tanto en el campo religioso como en el cívico. Su primera preocupación fue mantener un entendimiento armónico y cordial con la Iglesia y especialmente en lo relacionado con la educación. Dio orientaciones para que la materia de religión se introdujera en los planes de estudio, aunque dejó claridad para que se respetara y no se molestara a quienes no profesaban esta religión. Resaltó siempre el respeto a la moral cristiana católica y a la autoridad legítimamente constituida porque era defensora de los principios cristianos y la responsable de la enseñanza en materia religiosa.

También, es importante considerar que en el ideario de Rojas Pinilla estuvo siempre su interés y esfuerzos a favor de la mujer. Él comprendía que la mujer era abnegada por naturaleza, que tenía gran responsabilidad frente a la unidad familiar y, por lo tanto, debía ofrecerle todo su apoyo. Desde el momento que tomó legalmente posesión del cargo presidencial el 7 de agosto de 1954, con respecto a la mujer manifestó:

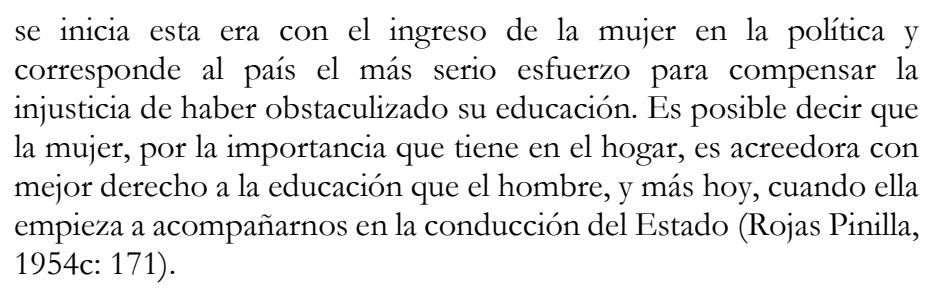

Con ese ideal se vinculó a la mujer al sistema educativo universitario, es decir, que la mujer desde la perspectiva política, cultural y social entraba a jugar papel preponderante en diversos campos de la actividad humana en Colombia. Pero, ante todo, se preparó el terreno para formarla como maestra y defensora de principios familiares, religiosos y cívicos. Este fue el tipo de formación que prácticamente concibió el gobierno de Rojas Pinilla y así se emprendió.

Entre las medidas educativas a favor de la mujer hay que destacar, en primer lugar, la consecución del derecho al voto cuyo significado fue de relevancia pedagógica. Así entró en escena con el ejercicio de la libertad, su participación en las decisiones políticas y su acceso a una mayor cultura. Aunque de manera desigual frente al hombre, logró igualdad teórica en programas escolares dentro del sistema educativo nacional y sobre todo se impuso para su beneficio y el de la sociedad un modelo de educación femenina que pudiera reconocerse por su valor y utilidad en beneficio de la cultura colombiana y del cambio de paradigmas sobre género.

\section{Conclusión}

Se puede anotar que, hacia la mitad del siglo XX, la universidad en Colombia fue el centro a donde confluían los problemas sociales, políticos, económicos, culturales, y como tal, debió contribuir a la solución de los mismos. En esas circunstancias, el papel 
La mujer y el papel de la universidad colombiana hacia mediados del siglo XX

de la universidad se enfocó a despertar el interés por la investigación y a abrir nuevos espacios para otros sectores de la población que hasta el momento habían sido marginados, como el caso de la mujer, cuyo ingreso a las aulas universitarias se dio para diversas carreras profesionales que le dieran la posibilidad de abrir un espacio en el campo laboral, además de haberse facilitado los medios para la creación de universidades femeninas que atendieran con exclusividad la formación de maestras.

\section{Referencias bibliográficas}

Chaves, José María (1957). La reforma universitaria en Colombia. Bogotá: Fondo Universitario Nacional.

Educación de la juventud (1954). Diana, 4.

Gaviria, Nicolás (1967). Filosofía e Historia de la Educación. Medellín: Bedout.

Le Bot, Ivon (1985). Educación e ideología en Colombia. Bogotá: La Carreta.

Muller de Ceballos, Ingrid (1992). La lucha por la cultura. La formación del maestro en una perspectiva internacional. Bogotá: Universidad Pedagógica Nacional.

Noticias de la Universidad (1953). Aulas, 9-10.

Posada, Jaime (1957). Universidad, democracia y país. Bogotá: Antares.

Rodríguez Rojas, José María (1963). Panorama de la educación colombiana. Medellín: Universidad de Antioquia.

Rojas Pinilla, Gustavo (1954a). Alocución Presidencial pronunciada con motivo de la Fiesta Nacional. 20 de julio de 1954. Mensajes y discursos presidenciales, 129-137.

Rojas Pinilla, Gustavo (1954b). Discurso pronunciado por el Señor presidente de la República Teniente General Gustavo Rojas Pinilla, ante la manifestación popular el día 7 de marzo de 1954, en Garzón - Huila. Mensajes y discursos presidenciales, 43-48.

Rojas Pinilla, Gustavo (1954c). Discurso pronunciado por el Teniente General Gustavo Rojas Pinilla al tomar posesión del cargo de la Presidencia, el 7 de agosto de 1954. Mensajes y discursos presidenciales, 151-180. 